\title{
ROUNDUP360
}

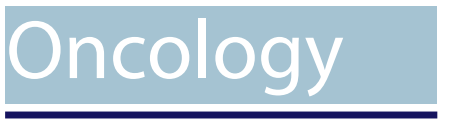

For other Roundups in this issue that cross-reference with Oncology see: Hip Roundup 3; Foot \& Ankle Roundup 7; and Trauma Roundup 4.

En bloc resection, irradiation and re-implantation

- There are widely reported case series describing the use of resection, irradiation and re-implantation for malignant bone tumours, although most are small series. Despite the obvious attractions of readily available anatomical block allograft, the technique has failed to gain widespread acceptance, mostly due to concerns regarding local recurrence and graft failure. Surgeons in Sydney (Australia) have been using the technique for over 15 years as part of their limb preservation algorithm. They undertook selective limb preservation surgery, performing an en bloc resection, immediate treatment with a single fraction of $50 \mathrm{~Gy}$ and synchronous re-implantation. The surgeons report a series of 101 serial patients, all of which were radiosensitive tumours, the majority of which were Ewing's sarcoma (35), osteosarcoma (37) and chondrosarcoma (20). There were similar results with Ewing's sarcoma and osteosarcoma patients. The authors report only a single local recurrence with Ewing's sarcoma and a five-year survival rate of $82 \%$. The osteosarcoma group had no local recurrences, but sadly five distant recurrences; However, they had a slightly higher five-year survival of nearly $86 \%$. While the study team reported similar five-year survival rates of $81 \%$ in the chondro- sarcoma group, they also report a high local recurrence rate of $20 \%$. This method was able to achieve limb salvage in $97 \%$ of patients, and for those with lower limb or pelvic disease over $80 \%$ were able to walk without aids at last follow-up.' The authors of this series present overall impressive five-year survivorship results and low local and distant recurrence rates. We would, however, question the safety of this technique in chondrosarcoma with one in five patients suffering local recurrence. Certainly here at 360 we wouldn't advocate this approach for patients with chondrosarcoma.

\section{Metastasis and osteosarcoma}

- Despite being the most common primary bone tumour, there is relatively little known about risks for metastasis in osteosarcoma. At presentation this makes a profound difference to both management strategy and expected outcomes for patients. With this in mind, a study team in lowa (USA) set about teasing out the risk factors for presentation with metastatic rather than distant spread at presentation. Using a database study model, the researchers used the Surveillance, Epidemiology, and End Results (SEER) Program database to identify the presence or absence of metastatic disease at the time of presentation. The study team also collated data on patient characteristics, socio-economic data and tumour characteristics using a combination of univariate analysis and a multivariate logistic regression model. The study cohort included
2017 patients presenting with high grade osteosarcoma, of which $23 \%$ (464 cases) had identified metastasis at the time of presentation. Factors identified leveraging an increased risk of metastasis at presentation include an age greater than 60 (OR 2.2), axial skeletal lesion (OR 2.5), and lower socio-economic status (OR 1.6). A more complex multivariant analysis of all three factors remained significant. There was a subset of patients $(n=1398)$ where tumour size information was recorded. Each additional $1 \mathrm{~cm}$ of tumour presentation size increased the odds of metastasis by $10 \%$ (OR 1.1). However, there was an interplay between tumour size and socioeconomic status. When patients with no tumour size were excluded, the socio-economic status was no longer a significant predictor of likelihood of tumour metastasis at presentation. Socioeconomic status is likely confounded by tumour size at presentation, with later presentation in patients of a lower socioeconomic group. ${ }^{2}$ While not setting the world alight for the originality of the design or earth shattering results, there is important information here derived from a large number of patients. Surgeons should be especially wary of older patients with bigger tumours and have a high index of suspicion for the presence of metastasis.

\section{Mobile spine and osteosarcoma}

- The mobile spine is not a common site for high grade osteosarcoma and even a large collaborative like the Cooperative Osteosarcoma Study Group in Stuttgart (Germany) were only able to report on the results of 20 patients over a 30-year period. They report a retrospective case series (Level IV evidence) of 20 patients presenting with high grade osteosarcoma since 1977 . The collaborators collated data concerning patient, tumour and treatment factors and have attempted to describe both the features and prognosis of such lesions. Patients presented at a median age of 29, with the majority of lesions located in the lumbar and thoracic spine. Around $85 \%$ of patients presented with isolated local disease. All patients in the series received surgery and adjuvant chemotherapy, with $65 \%$ also undergoing radiotherapy. This treatment yielded 12 patients completely in remission with eight cases of recurrence (five local recurrence, one distant and two with local recurrence and metastasis). Of those with recurrence, six died and two (both having received radiotherapy) survived. Of those patients with initial remission, three subsequently had recurrence (two local, one metastatic) and died. The 11 survivors in this series were followed for an average of over eight years, yielding a five-year survival of $60 \%$ ( $43 \%$ event free). An odds of survival analysis demonstrated young age, lack of metastatic disease at diagnosis and complete remission as predictors of survival. ${ }^{3}$ While a rare diagnosis that often presents late, these authors have demonstrated that with radical 
surgery and radiotherapy, acceptable survival figures comparable with those seen with appendicular osteosarcoma can be achieved.

\section{Denosumab miraculous for GCT}

- The giant cell tumour of bone (GCT) is a rare, borderline malignant lesion which has the ability both to metastasise and recur (recurrence is often particularly aggressive). There is currently no standard medical or chemotherapy regime available for treatment of GCTs. An international study team, led by surgeons in Santa Monica (USA) has reported on a phase II efficacy study of denosumab for the treatment of these lesions.

The study has a complex design investigating three distinct cohorts of patients; those with unsalvageable GCT; those with salvageable GCTs but high morbidity risk associated with surgery; and finally a cohort of patients from a previous RCT who continued their allocated treatment. The study design was of an open label parallel-group phase II trial of patients with confirmed GCT and radiographically active disease. Treatment was with a $120 \mathrm{mg}$ of $\mathrm{S} / \mathrm{C}$ denosumab every four weeks with an initial loading phase. The investigators recruited 282 patients, of whom ten were adolescent over a two and a half-year period. The primary end point was safety, and there were a range of adverse side effects occurring in $9 \%$ of patients $(n=25)$. The investigators reported an incidence of osteonecrosis of the jaw (1\%), hypocalcaemia (5\%) and hypophosphataemia (3\%). Other less serious side effects included anaemia, back pain, and pain in extremities, each of which occurred in three patients (1\%). Perhaps the most interesting findings of the study were that in all cohorts studied, the intervention was remarkably effective. In the unresectable group, $96 \%$ $(n=163 / 169)$ had no disease progression, and in the co-morbid group $74 \%$ $(n=74 / 100)$ required no surgery, and $62 \%$ of those having surgery underwent a less morbid procedure than initially planned. ${ }^{4}$ The adverse events

reported here in this study were in line with the known side effect profile of denosumab, which represents a new treatment option for patients with GCT. The remarkable thing to us, here at 360 , is that here appears to

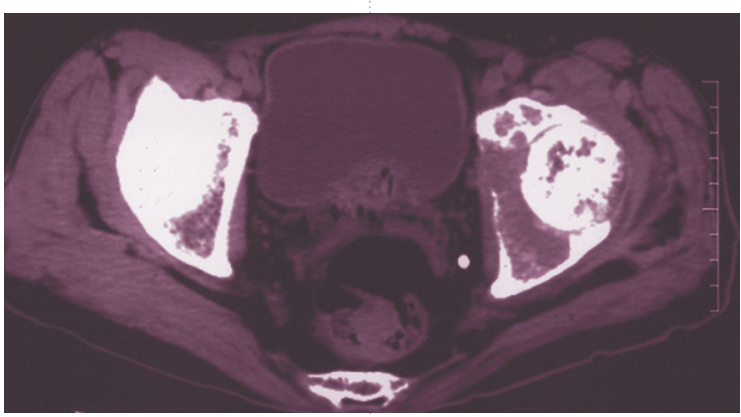

be a treatment that is more effective than the majority of drugs out there and certainly must be one of the most effective interventions for any type of tumour. The question that does occur is that it may need to be continued for life, and would it be an effective adjuvant or neo-adjuvant treatment for surgical treatments. Certainly this represents one of the most important surgical oncology papers we have seen here at 360 this year.

\section{Fevers, megaprostheses and sarcomas}

- The so-called 'megaprosthesis' has become a staple of limb salvage surgery. Throwing caution to the wind, tumour surgeons the world over are now embarking on ambitious surgical reconstructions ranging from the total femur replacement to pelvic replacements. While limb salvage of this type is known to provide excellent functional outcomes, the spectres of both arthroplasty and tumour complications raise their heads. Post-operative pyrexia is common in major surgery, but presents a diagnostic dilemma in megaprosthesis surgery. Given the serious implications of deep infection and pyrexia, making a diagnosis can be a great dilemma. Researchers in Seoul (Korea) set out to establish the incidence of post-operative pyrexia and the clinical significance after megaprosthetic surgery. The study team were able to muster a 71-patient retrospective cohort study (Level IV evidence), having previously undergone megaprosthesis reconstruction for lower-extremity osteosarcoma. There was no evidence in any patient of pre-operative

concomitant infection. Patients were followed up to nearly five years after their surgery and were, on average, 24 years old pre-operatively. There was a $7 \%$ post-operative infection rate within the series, but an $87 \%$ incidence of post-operative pyrexia (defined as $>38^{\circ} \mathrm{C}$ ), which in the vast majority of cases settled by day five post-operatively. The researchers were unable to establish any association between the presence of a post-operative fever and wound infection. However, in patients with clinically proven infection, swinging pyrexias and prolonged fevers (beyond day five) were noted. ${ }^{5}$ Like so many things in clinical medicine, the authors of this review recommend clinical examination and review postoperatively - sensible advice.

\section{PET and prognosis}

- Positron emission tomography (PET scanning) uses a tracer (usually labelled glucose) and a gamma camera to detect the metabolic activity of a tissue. This is usually coupled with a CT scan and has become a more and more widely used imaging modality, particularly in all types of oncological medicine (where metabolic activity can be a useful marker of tumour burden and turnover). This technology is rarely applied to musculoskeletal tumours, and researchers in Montreal (Canada) have taken the opportunity to establish if necrosis (as quantified by a PET/
CT scan) is indicative of prognosis in sarcoma patients. The research team used the PET/CT technique in 66 patients, all diagnosed with limb or pelvic sarcomas between 2004 and 2009. They designed a study to establish the prognostic value of the tumour standardised uptake values (SUVmax), the presence of necrosis and the volume of necrosis. Followup data were used to establish the overall survival and event free survivals. A proportional hazards model was then used to estimate hazards ratios. This inclusive study included soft tissue (70\%), bone (24\%) and 'other' sarcomas with a mean followup of over 33 months. During the duration of the study, overall survival was 59\%. There was a statistically significant relationship between prognosis and necrosis, with survivals of $96 \%, 65 \%$ and $38 \%$ at two years for patients with no necrosis, some necrosis and more than 50\% necrosis respectively. ${ }^{6}$ This is a really encouraging study with the potential of a new independent prognosticator for all types of musculoskeletal tumour. This study raises the exciting prospect for us, here at 360 , that PET/ CT may also be useful to monitor the efficacy of chemotherapy regimes.

\section{Canine sarcomas not so different?}

- By way of proof that there is no paper that escapes the beady eyes of our editorial board here at 360 , we would draw your attention to a paper from the world of veterinary medicine. Vets in Urbana (USA) have investigated the bone turnover in dogs with appendicular osteosarcoma. The study was designed to establish if serum bone-specific alkaline phosphatase (BALP) activity is a negative prognostic factor for dogs with appendicular osteosarcoma (OSA). However, for us here at 360 , there is a much more important side to this study. The veterinary surgeons recruited 96 dogs with appendicular osteosarcoma. They investigated the expression and membranous release of BALP to establish any potential correlation between serum 
BALP activity and primary tumour size. Secondary outcomes were the observed changes in BALP in dogs developing visceral OSA metastases. The vets established that BALP expression was not associated with phenotype but rather, with the cell density. BALP activity was a marker for both tumour size and metastatic lesions.? This finding is extremely interesting as a similar relationship between BALP and tumour size and metastasis is seen in humans. These findings of similar metabolic activity are highly suggestive that a canine model of osteosarcoma is an ideal animal model of disease.

\section{Bone cement and giant cell tumours}

- For those patients who are still undergoing surgery for giant cell tumours (with or without denosum$a b)$, one of the largest unanswered questions is what to put in the defect: bone cement or bone graft? A review group in Shanghai (China) set out to review the literature and establish the current 'state of the art' approach. They designed a careful meta-analysis and systematic review with the aim of reviewing the efficacy of PMMA bone cement and allogenic bone graft following intralesional curettage for GCTs. A thorough search of the indexed literature was followed by a fairly standard methodology including pooled risk ratios and 95\% confidence intervals ( $\mathrm{Cls}$ ) for local recurrence risks using a fixedeffects method (rather than the more complex random effects model). The study team identified 1690 relevant articles via search terms, of which six studies fulfilled the inclusion criteria (1293 patients). Patients undergoing bone graft alone suffered higher recurrence rates than PMMA-treated patients (RR 2.09), a result which was mirrored in patients also receiving adjuvant treatments (RR 1.66). ${ }^{8} \mathrm{It}$ certainly appears to us here at 360 that based on these results PMMA is the treatment of choice for treatment of GCT.

\section{REFERENCES}

1. Hong AM, Millington $S$, Ahern V, et al. Limb preservation surgery with extracorporeal irradiation in the management of malignant bone tumor: the oncological outcomes of 101 patients. Ann Oncol 2013; Epub ahead of print.

2. Miller BJ, Cram P, Lynch CF, Buckwalter JA Risk factors for metastatic disease at presentation with osteosarcoma: an analysis of the SEER database. J Bone Joint Surg [Am] 2013;95-A:891-8

3. Zils $K$, Bielack $S$, Wilhelm $M$, et al. Osteosarcoma of the mobile spine. Ann Oncol 2013;24:2190-5

4. Chawla S, Henshaw R, Seeger L, et al.
Safety and efficacy of denosumab for adults and skeletally mature adolescents with giant cell tumour of bone: interim analysis of an open-label, parallelgroup, phase 2 study. Lancet Oncol 2013;14:901-8.

5. Kim W, Han I, Lee SA, Cho HS, Kim HS. Febrile response following megaprosthesis replacement for primary bone sarcoma. Orthopedics 2013;36:695-9.

6. Rakheja R, Makis W, Tulbah R, et al. Necrosis on FDG PET/CT correlates with prognosis and mortality in sarcomas. AJR Am J Roentgenol 2013|;201:170-7.

7. Sternberg RA, Pondenis HC, Yang X, et al. Association between absolute tumor burden and serum bone-specific alkaline phosphatase in canine appendicular osteosarcoma. J Vet Intern Med 2013;27:955-63.

8. Zuo D, Zheng L, Sun W, et al. Contemporary adjuvant polymethyl methacrylate cementation optimally limits recurrence in primary giant cell tumor of bone patients compared to bone grafting: a systematic review and meta-analysis. World $J$ Surg Oncol 2013;11:156. 